\title{
Perception of visual temporal patterns by deaf and hearing adults
}

\author{
CAROL BERGFELD MILLS \\ American Institutes for Research, Washington, DC
}

\begin{abstract}
The performance of deaf and hearing college students was compared in a same-different task involving visual temporal patterns. The results showed equivalent performance for the two groups. For both deaf and hearing subjects, hierarchically simple patterns were easier than more complex patterns, which is consistent with a model of temporal pattern perception proposed by Martin (1972).
\end{abstract}

Hearing is commonly assumed to be the main sense through which temporal information is perceived. Fraisse, for example, stated that "hearing is the main organ through which we perceive change: it is considered as the 'time sense' ,' (1963, pp. 82-83). A number of theorists (e.g., Gibson, 1969; Hebb, 1949) have suggested that experience plays a critical role in the development of perceptual skills. According to these views, it might be expected that the ability to perceive temporal information may depend on the amount of auditory stimulation experienced. If auditory stimulation is critical in the development of the ability to perceive temporal information, then people who have had no or very limited auditory experience-the congenitally deaf-should not perceive temporal information as well as people with normal auditory experience.

It is also possible that auditory experience per se may not be critical to the ability to perceive temporal information. This ability may be a universal one that is innate. Alternatively, this ability could develop as a result of nonauditory experience. For example, some motor movements such as breathing and walking are rhythmic, and the experience of these movements may facilitate the perception of rhythm. If the ability does not depend on auditory experience, then no differences would be expected between deaf subjects and hearing subjects. The purpose of this paper is to report a study conducted to compare deaf and hearing subjects on their abilities to perceive temporal information. More specifically, this study was concerned with subjects' abilities to perceive differences in temporal duration(s) of an element or elements in a pair of visual patterns. The ability to perceive the temporal changes will be called "timing sensitivity."

The research was conducted while the author was a postdoctoral fellow in the Psychology Department at Gallaudet College and was supported by a grant from the Office of the Coordinator of Faculty and Graduate Student Research at Gallaudet College. Computer services were provided in part by the Computer Science Center at the University of Maryland. The help of Carla Banas in tabulating and analyzing the data is appreciated. For reprints, write C. B. Mills, American Institutes for Research, 1055 Thomas Jefferson Street, NW, Washington, DC 20007.
Previous research comparing the abilities of hearing and deaf individuals to perceive and respond to temporal changes has been inconclusive. Sterritt, Camp, and Lipman (1966) found that hearing impaired subjects between 3 and 7 years old did not reproduce visual, temporal patterns as well as subjects of the same age with normal hearing. Sterritt et al. obtained the same results with abovethreshold auditory patterns. They concluded that the auditory deprivation experienced by hearing-impaired children produces deficiencies in temporal abilities which are not specific to the auditory modality. However, the Sterritt et al. study was criticized by Rileigh and Odom (1972) on the grounds that the patterns were constructed in such a way that nontemporal relationships between the pattern elements could have been used to perform the task.

Rileigh and Odom (1972) studied three aspects of the reproduction of visual patterns for both deaf and hearing subjects. Ten-year-old deaf subjects were less accurate than hearing 10-year-olds in reproducing the total duration of visual patterns, but 15-year-old deaf and hearing subjects did not differ. The best performance in reproducing the number of beats was shown by older hearing subjects on shorter patterns, and the poorest performance was by younger hearing subjects on longer patterns. Hearing status had no effect on accuracy of rhythm reproduction, the measure of greatest interest for the present paper. From these findings, it is difficult to draw any general conclusions concerning the effect of hearing status on the ability to perceive or reproduce temporal patterns. The task used by Rileigh and Odom (1972), as well as by Sterritt et al. (1966), adds to the difficulties in interpreting their data, because the task involved both the ability to perceive a pattern and the ability to reproduce it. If the deaf perform more poorly, it is not clear whether poor performance is due to a deficiency in perception or a deficiency in (re)production.

Using a measure of perception of temporal patterns that did not involve reproduction, Kracke (1975) found no differences between subjects ( 8 to 15 years old) with profound hearing losses and their normal-hearing counterparts. In the Kracke study, same-different judgments were obtained for pairs of cutaneous patterns and for pairs of 
above-threshold auditory patterns. On the basis of the Kracke results, it might be concluded that auditory experience is not critical to the development of timing sensitivity. However, as Kracke suggested, the failure to obtain differences between the hearing and deaf subjects may have been the result of a ceiling effect.

The present research differed in a number of ways from the previous studies comparing deaf and hearing subjects. The subjects were college students rather than younger children. The present study used a perception task similar to Kracke's in which subjects made same-different judgments about pairs of patterns; however, visual patterns rather than auditory or cutaneous ones were used. Pilot testing was done to establish guidelines for designing patterns that were difficult enough so that a ceiling effect would not occur.

Another purpose of the present study was to test predictions derived from a model of temporal pattern perception (Martin, 1972). According to Martin's model, temporal patterns are perceived and encoded in a hierarchical manner, which can be formalized in terms of rhythm trees. It would be expected, on the basis of this model, that temporal patterns which have simpler hierarchical structures should be easier to perceive than those which have more complex structures. For example, patterns which can be segmented into two parts of equal duration should be easier to perceive than those which cannot be segmented into two parts, because those patterns, which can be segmented would "fit" a hierarchical rhythm tree. Also patterns with fewer levels in a hierarchical tree should be easier to perceive than those with more complex, multilevel structures. The present experiment studied the perception of visual temporal patterns to determine whether differences in performance (if any) between simple and complex patterns were the same for deaf and hearing subjects.

\section{METHOD}

\section{Experimental Materials}

The stimuli were 64 pairs of visual patterns. All of the patterns consisted of eight Xs presented on a TV screen. The Xs of a given pattern were added sequentially to the screen. Only the timing of the appearance of the Xs varied between patterns; the left-to-right order of presentation, the spacing between the Xs, and the number of Xs all were the same in each pattern. The interval between the presentation of Xs (or until the whole pattern disappeared if it was the eighth $X$ ) was either 25,50 , or 100 centiseconds ( $\operatorname{csec}$ ) with at least one of each of the three durations present in each pattern. Each pattern of eight Xs lasted a total of $400 \mathrm{csec}$ (with an exception mentioned later). The duration of the first $X$ was always $50 \mathrm{csec}$.

For example, the Xs in a given pattern could have the following durations: $50,25,25,100,50,25,25,100 \mathrm{csec}$. For this example, the first $X$ appears on the left-hand side of the screen. Fifty csec later, the second $X$ is added to the screen and appears to the right of the first $X$. Then $25 \mathrm{csec}$ later, the third $X$ appears to the right of the second $X$. The fourth $X$ appears $25 \mathrm{csec}$ later to the right of the third $X$, and so on for the remaining Xs. For this example, the eighth $X$ stays on the screen for $100 \mathrm{csec}$, and then it and the other Xs disappear from the screen.

Thirty-two different initial patterns were generated. Half of these initial patterns had simpler hierarchical structures (Martin, 1972) and will be called simple patterns. The other half had more complex structures and will be called complex patterns. The simple patterns were simple in that the onset of one of the Xs in the patterns always divided the patterns into two equal time segments of $200 \mathrm{csec}$, enabling their "fit" to a rhythm tree. The complex patterns could never be divided into two equal time segments by the onset of an $\mathrm{X}$. In addition, the simple patterns had simpler structures than the complex ones in that Xs with 25$\operatorname{csec}$ durations always occurred in pairs so that the duration of the pair was equal to that of an $\mathrm{X}$ with a longer duration $(50 \mathrm{csec})$. As a result, for the simple patterns, fewer levels in a rhythm tree were required to "ffit" the patterns. In all but three of the complex patterns, Xs with 25csec durations did not occur in pairs.

Each initial pattern was used twice: once in a pair in which the second pattern was the same as the first (same pairs) and once in a pair in which the second pattern was different from the first (different pairs). An equal number of pairs in which the first and second patterns differed were randomly assigned to have either one, two, three, or four timing differences.

For the pairs of patterns that differed by only one timing difference, 25 or $50 \mathrm{csec}$ was added to or subtracted from one element of the second pattern in the pair (i.e., the total time constraint of $400 \mathrm{csec}$ was violated). For the pairs of patterns that differed by two timing differences, a duration in serial positions 2 to 7 in a pattern was randomly selected, and that duration and the next one which differed were switched within the second pattern of the pair. For the pairs of patterns that differed by three timing differences, one duration in positions 2 to 6 was randomly selected, and it and the following two durations which differed were permuted. For the pairs of patterns that differed by four timing differences, two nonadjacent durations in positions 2 to 7 were randomly selected, and each of those durations was switched with the next one that differed.

The visual patterns were made with the aid of a PDP-12 computer. The timing information for the pairs of patterns were entered into the computer in four blocks of 16 pairs each. In each block, half of the pairs were simple and half were complex. In addition, half were same and half were different, with the constraint that there were two pairs each with one, two, three, or four timing differences per block.

In order to make the experimental stimuli, the pairs of patterns were displayed by the computer on a cathode ray tube (CRT). The pairs were presented with $1 \mathrm{sec}$ between the time when the eight Xs of the first pattern of a pair disappeared and the first $X$ of the second pattern appeared. When all eight Xs of the second pattern disappeared, the word "RESPOND" appeared on the CRT for $7 \mathrm{sec}$. The output of the CRT was recorded on a video tape, which was used to present the stimuli to subjects.

In addition to the 64 experimental pairs of patterns, 4 practice pairs, 2 same and 2 different, were constructed and recorded in the same manner.

\section{Procedure}

Subjects were run in groups of up to 10 persons. First, all subjects were asked to provide answers to a few biographical questions. For the hearing subjects, the questions concerned musical training, gender, and age. For the deaf subjects, the questions concerned age of deafness onset, length of sign language experience, gender, and age.

The instructions for the hearing subjects were presented orally, and the instructions for the deaf subjects were presented in simultaneous communication (sign and voice). Subjects were told that they would see pairs of visual patterns on the TV and that their task was to determine whether the timing of the Xs in each pair of patterns was the same or different. Then the practice pairs were played on a video monitor. If questions arose, the practice pairs were replayed. Then the four blocks, each containing 16 pairs of patterns, were played on the video monitor with a brief rest between blocks. After both patterns of the pair were presented, subjects indicated their responses by circling an $S$ for same or a $\mathrm{D}$ for different on their answer sheets.

\section{Subjects}

The subjects were 44 deaf students at Gallaudet College and 50 hearing students at the University of Maryland, College Park. Both groups of subjects participated in the experiment in order to gain extra credit for their psychology classes.

The deaf subjects, who will be called the prelingually deaf (average 
age $=22.5$ years), reported either that they were congenitally deaf $(n=26)$ or that they had lost their hearing by the age of 2 years $(n=9)$. Nine other deaf subjects, who will be called the later-onset deaf (average age $=24.1$ years), lost their hearing later in life (mean age of hearing loss $=9.7$ years; range $=3.5$ to 18 years). All of the prelingually deaf subjects and seven of the nine later-onset deaf subjects reported using American Sign Language as their primary means of communication. While the audiograms for the individual deaf subjects were not available, their auditory experience was qualitatively and quantitatively less than that of the hearing subjects. All deaf subjects had at least a $60-\mathrm{dB}$ hearing loss in the better ear since that was the criterion for admission to $\mathrm{Gal}$ laudet College. Four additional deaf subjects were run but were not included in any of the data analyses because they failed to follow instructions.

The hearing subjects had an average age of 19.3 years. Four additional hearing subjects were run but were omitted from the data analyses because they did not follow instructions.

\section{RESULTS}

The percent of correct judgments for simple and complex patterns was calculated for each subject. The mean percent correct for each subject group is shown in Table 1. An unweighted means solution ANOVA for groups of unequal size (Winer, 1971) was conducted on the percent correct judgments. This analysis revealed that more correct judgments were made for simple patterns $(70.9 \%)$ than for complex ones $(66.4 \%)[\mathrm{F}(1,83)=11.63, \mathrm{p}<$ $.01]$. The effect of subject group was not significant, and the subject group did not interact with the simple-complex factor. A test for homogeneity of variance showed that there were no significant differences between deaf and hearing groups in the variance for the percent correct judg$\operatorname{ments}[\operatorname{Fmax}(2,50)=1.18]$.

To determine whether the simple-complex factor had a similar effect for both same and different pairs, the percent of correct simple and complex judgments was calculated separately for those pairs. These data showed that for both same and different pairs, more correct judgments were made for simple patterns than for complex ones for both subject groups. The mean percent of correct judgments for simple and complex patterns in the same pairs for all subjects combined were $75.6 \%$ and $71.1 \%$ respectively. The corresponding means for the different pairs were $66.2 \%$ and $61.6 \%$. Thus, the magnitude of the simple-complex factor was similar for the same and different pairs and, therefore, cannot be attributed to the way the different pairs were constructed.

To test whether the amount of auditory experience of the deaf affects their performance, the percent of correct judgments was calculated for the nine later-onset deaf subjects. That percent was the same as the percent for the

Table 1

Percent of Correct Judgments

\begin{tabular}{lcccc}
\hline & \multicolumn{4}{c}{ Pattern Type } \\
\cline { 2 - 5 } Subject Group & $\mathrm{N}$ & Simple & Complex & Combined \\
\hline Prelingually Deaf & 35 & 70.2 & 66.3 & 68.2 \\
Hearing & 50 & 71.5 & 66.4 & 68.9 \\
Combined & 85 & 70.9 & 66.4 & \\
\hline
\end{tabular}

prelingually deaf (68.2\%), indicating no effect of amount of experience.

For the hearing subjects, analyses were performed to determine whether auditory experience in the form of musical training had any effect on performance on the visual patterns. For this purpose, for the hearing subjects who reported receiving musical training on the biographical questions, a correlation was calculated between the number of years of musical training reported and the number of correct responses on the visual patterns. No significant relationship was found $[\mathrm{r}(26=+.20]$ for the range of musical training in this sample (1 to 8 years).

\section{DISCUSSION}

Deaf and hearing subjects were compared in order to determine what effect auditory experience has on the ability to perceive visual temporal patterns. In addition, the effect of pattern structure on perception was studied by comparing performance on hierarchically simple and more complex patterns.

The results showed that for deaf and hearing groups, simple patterns were easier to perceive than complex ones. There was no evidence that auditory experience affects performance: The two groups did not differ in the number of pairs of patterns correctly judged. Also there was no difference in performance for the prelingually deaf and the later-onset deaf, and there was no relationship between the amount of musical training, for the hearing subjects who had musical training, and their performance on the visual patterns.

It might be postulated that the reason there was no difference between hearing and deaf subjects is that some of the deaf subjects had residual hearing and, therefore, had auditory experience. However, the deaf subjects' auditory experience was clearly qualitatively and quantitatively different than that of hearing subjects. A second reason that might be suggested for the lack of a difference between hearing and deaf subjects is that deaf subjects are really poorer at timing tasks, but have a compensating visual ability. In other words, the deaf might be able to perform visual tasks better than the hearing. If the deaf perform temporal tasks more poorly than the hearing, but perform visual tasks better than the hearing, the result might be performance equivalent to the hearing. The idea of the compensation of the visual sense for the deaf has been explored in a number of studies; however, there is little evidence to support the idea (Reynolds, 1978). A tradeoff then between visual compensation and deficiencies in temporal abilities appears to be an unlikely interpretation for the present results with hearing and deaf subjects. A more reasonable interpretation of the equivalent performance of the hearing and the deaf subjects appears to be that auditory experience is not critical to the development of timing sensitivity.

The results are consistent with the hierarchical model of temporal pattern perception proposed by Martin (1972). The hierarchically simple patterns were easier than more complex patterns for both groups of subjects. This was found for both the same and different pairs of patterns indicating that the simple-complex effect was not due to the effects of the number of duration differences or any other factors which might affect performance on the different pairs. These findings suggest that the simpler the hierarchical structure of information on a temporal dimension, the easier it is to perceive.

Visual patterns used in the present study seem to serve as a useful tool for investigating temporal perception. Based on the performance of 56 additional hearing subjects, the reliability of the visual patterns as a measure ( $r=+.64$, split-half) was identical to that reported for a commonly used auditory measure of rhythm, the Seashore measure (Seashore, Lewis, \& Saetveit, 1960). Also based on the performance of these hearing subjects, there is a positive relationship $[\mathrm{r}(54)=+.43$, $\mathrm{p}<.001$ ] between performance on the visual patterns and the Seashore measure. That relationship is stronger $[\mathrm{r}(54)=+.64]$ when taking the reliabilities of the measures into account (Guilford, 1954). This later finding indicates that to some extent the two measures assess the same 
abilities or processes. The results of an earlier study (Mills \& Jordan, 1980) also provide evidence of predictive validity for the visual pattern measure. Mills \& Jordan (1980) showed that hearing adults who performed better on visual patterns like those used in this study also obtained higher semester grades in sign language courses $[\mathrm{r}(98)=+.35$, $\mathrm{p}<.001]$. Thus, the visual patterns used in the present study and visual patterns like these may be useful for exploring further questions about temporal pattern perception, particularly in the deaf for whom auditory patterns may not always be appropriate.

\section{REFERENCES}

Fraisse, P. (1963). The psychology of time. New York: Harper \& Row. Gibson, E. J. (1969). Principles of perceptual learning and development. New York: Appleton.

GuILFORD, J. P. (1954). Psychometric methods (2nd ed.). New York: McGraw-Hill.

HeBb, D. O. (1949). The organization of behavior. New York: Wiley.

KRACKE, I. (1975). Perception of rhythmic sequences by receptive aphasic and deaf children. British Journal of Disorders of Communication, 10, 43-51.
MARTIN, J. G. (1972). Rhythmic (hierarchical) versus serial structure in speech and other behavior. Psychological Review, 79, 487-509.

Mills, C. B., \& JoRDAN, I. K. (1980). Timing sensitivity and age as predictors of sign language learning. Sign Language Studies, 26, 15-28.

REYNOLDS, H. N. (1978). Perceptual effects of deafness. In R. D. Walk \& H. L. Pick, Jr. (Eds.), Perception and experience. New York: Plenum.

Rileigh, K. K., \& Odom, P. B. (1972). Perception of rhythm by subjects with normal and deficient hearing. Developmental Psychology, 7, 54-61.

SeAShORE, C. E., Lewis, L., \& SAETVeit, J. G. (1960). Seashore measure of musical talent. New York: The Psychological Corp.

Sterritt, G. M., CAMP, B. W., \& Lipman, B. S. (1966). Effects of early auditory deprivation upon auditory and visual information processing. Perceptual \& Motor Skills, 23, 123-130.

WINER, B. J. (1971). Statistical principles in experimental design (2nd ed.). New York: McGraw-Hill.

(Manuscript received for publication April 2, 1985.) 